\title{
Plain and Contrast Enhanced CT Imaging Findings of a Rare Case of IVC Pseudoaneurysm
}

\author{
Aruna Devi*, Rama Kumari, Sandeep Mahapatra \\ Nizam's Institute of Medical Sciences, Hyderabad, India \\ Email: *arunabilu@yahoo.co.in, testinet@yahoo.co.in, ramanuthalapati@yahoo.in, sanusandeep@gmail.com
}

How to cite this paper: Devi, A., Kumari, R. and Mahapatra, S. (2018) Plain and Contrast Enhanced CT Imaging Findings of a Rare Case of IVC Pseudoaneurysm. Open Journal of Internal Medicine, 8, 84-88. https://doi.org/10.4236/ojim.2018.81009

Received: August 11, 2017

Accepted: March 13, 2018

Published: March 16, 2018

Copyright $\odot 2018$ by authors and Scientific Research Publishing Inc. This work is licensed under the Creative Commons Attribution International License (CC BY 4.0).

http://creativecommons.org/licenses/by/4.0/

\begin{abstract}
Inferior vena cava pseudo aneurysms are rare clinical entities with an uncertain natural history due to its rarity and limited follow up information. IVC injuries are managed according to the patient's presentation and hemodynamic status. This case report describes clinical presentation of a 30 years old man with history of fall from height, infra renal IVC pseudoaneurysm found with FAST and CT scan abdomen, our treatment plan and his clinical outcome.
\end{abstract}

\section{Keywords}

CT, Duplex Sonography, IVC Pseudoaneurysm, Predictors of Mortality, Treatment Options

\section{Introduction}

Blunt injury to the inferior vena cava is rare, ascribable for approximately $30 \%$ $40 \%$ of abdominal vascular injuries [1]. Sudden deceleration causes shear forces on vessels. Inferior vena cava injuries are managed according to the patient's hemodynamic status.

\section{Case Report}

A 30 year old man was referred to our hospital from a referring hospital after fall from height. At the time of admission patient was hemodynamically stable with normal vitals but with diffuse pain abdomen and a positive focused assessment with sonography in trauma (FAST) examination.

USG showed contained extravascular collection lying posterior to and in contact with infra renal segment of IVC. Duplex Sonography showed swirling flow in the sac and connecting tract with IVC (Figure 1 \& Figure 2). 
CT scan abdomen was obtained, which showed small contusion in segment V of liver, and irregularity of infra renal IVC with contained contrast extravasation consistent with IVC pseudoaneurysm (Figure 3-5), partial enhancement of IVC distal to the pseudoaneurysm and left common iliac vein consistent with partial thrombosis (Figure 6), absent enhancement of left external iliac vein, common femoral, visualized segments of superficial and profunda femoral veins consistent with complete thrombosis. Mild bilateral pleural collection, mild to moderate retroperitoneal hematoma (in right perinephric space, pericaval region, along both iliac vessels, and along psoas muscles). Minimal intraperitoneal free fluid in the Morison's pouch. Fracture left transverse process of L1, L2 and L4 vertebra, right transverse process of L3 vertebra. Observation of these lesions

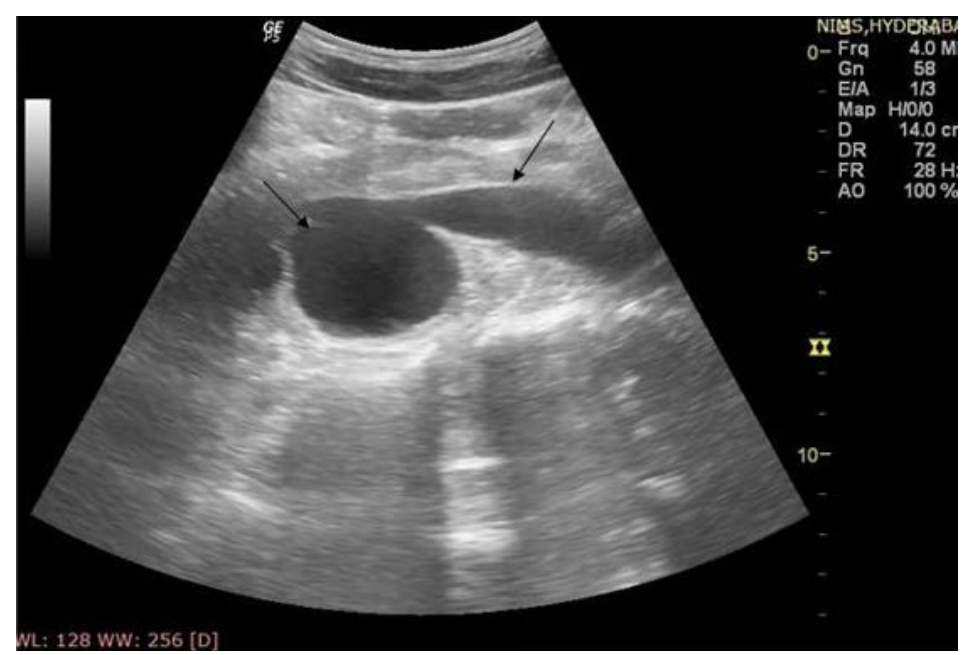

Figure 1. Duplex sonography showing contained extra vascular collection iying posterior to and in contact with infra renal segment of IVC. Adjacent IVC segment is attenuated.

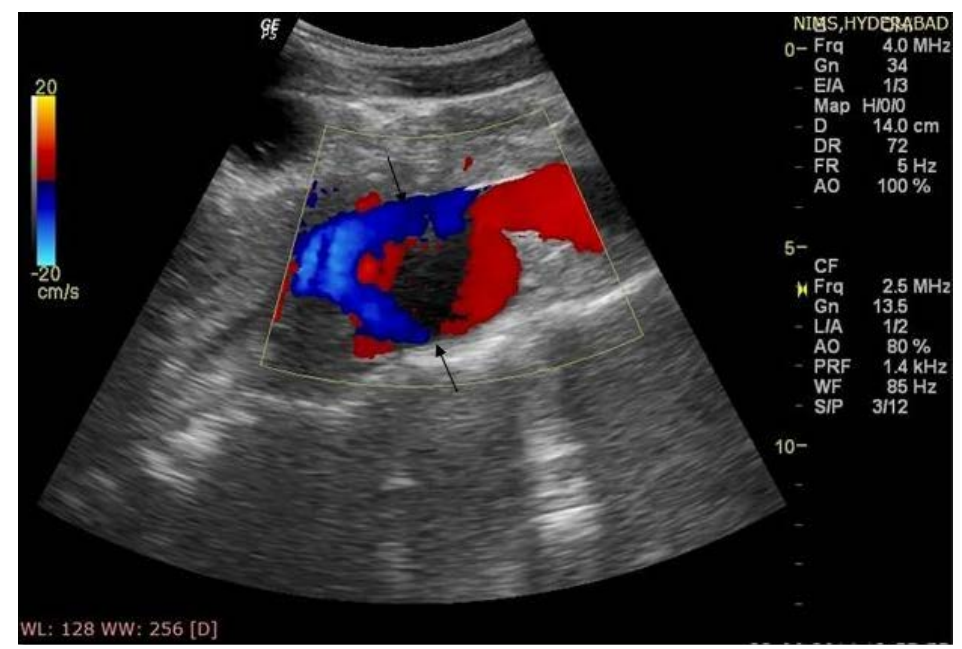

Figure 2. Duplex sonography showing contained extra vascular collection iying posterior to and in contact with infra renal segment of IVC. Sac is connected to IVC through a track. Sac showed swirling flow within. adjacent IVC segment is attenuated. 


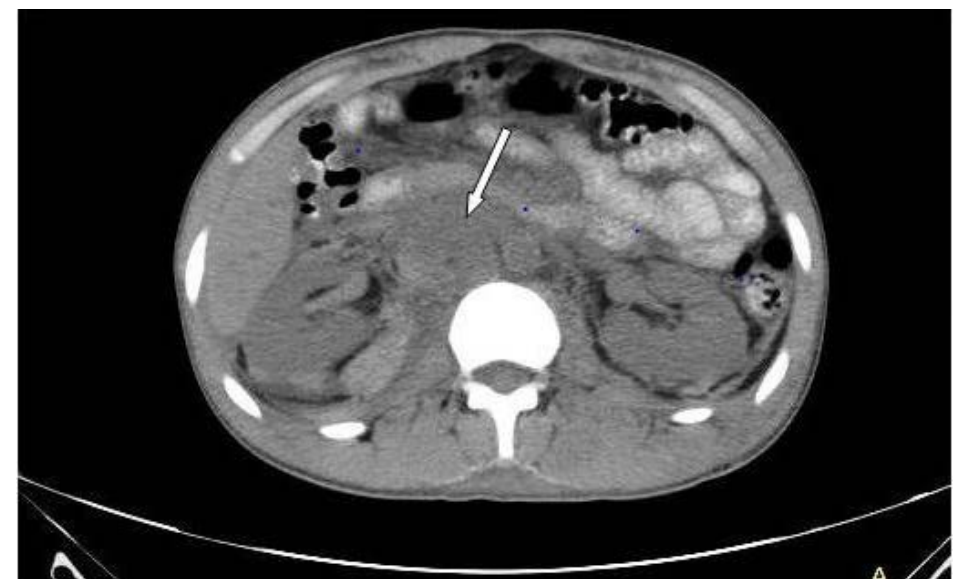

Figure 3. Plain CT abdomen axial image at the level of renal hilum showed irregular mixed density collection in the retro-peritoneum (at pericaval region tracking into right perinephric space) consistent with haematoma.

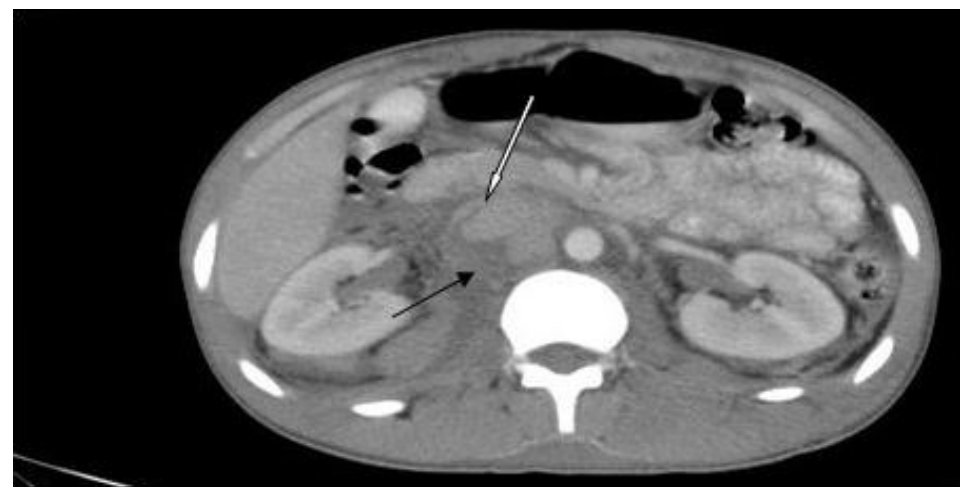

Figure 4. Contrast enhanced CT axial image at the level of renal hilum showed contained contrast extravasation consistent with Pseudoaneurysm into the retroperitoneum (posterior and lateral to the IVC which has extended into the right perinephric space. Adjacent IVC is attenuated and lifted anteriorly.

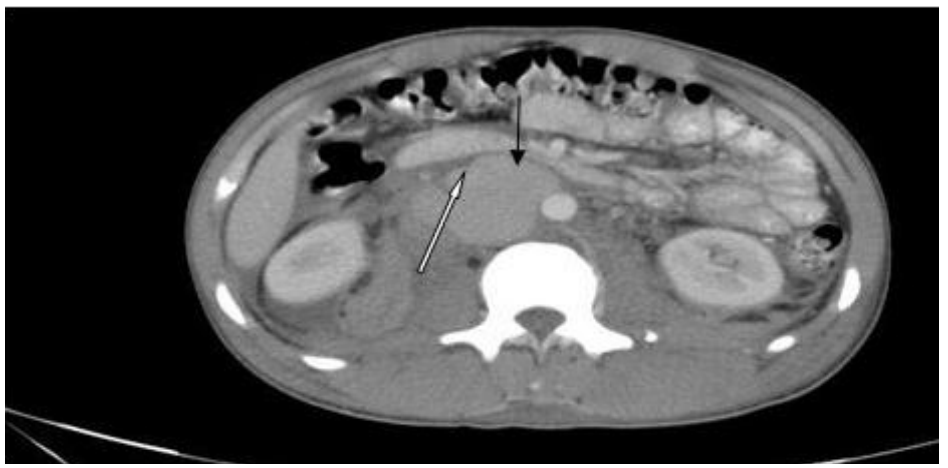

Figure 5. Contrast enhanced CT axial images at the level of renal hilum showed contained contrast extravasation consistent with pseudoaneurysm into the retroperitoneum (posterior and lateral to the IVC which has extended into the right perinephric space. Adjacent IVC is attenuated and lifted anteriorly. 


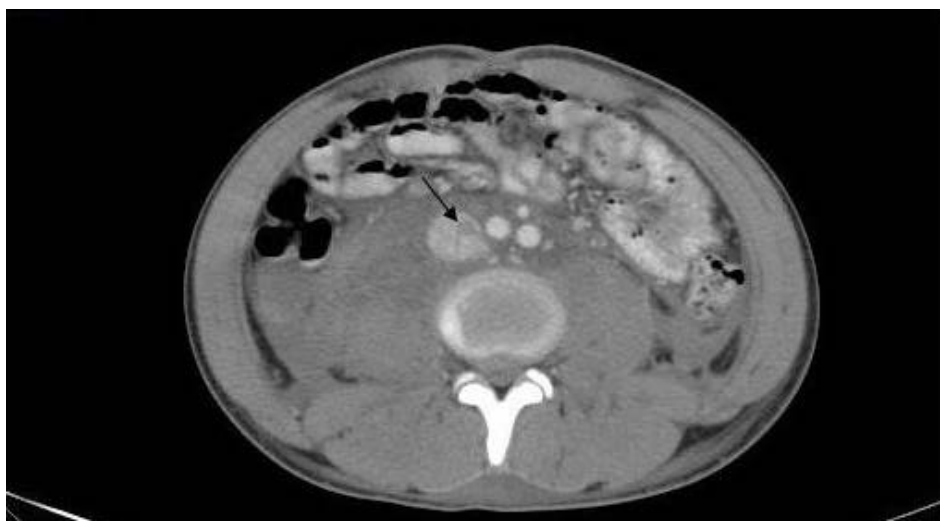

Figure 6. Contrast enhanced CT abdomen axial image caudal to the pseudoaneurysm sac showed partial filling defect in the IVC consistent with partial thrombosis.

felt to be the safest course of action in this patient, as described in literature, who was hemodynamically stable at the time of diagnosis.

A repeat CT venogram performed 10 days after injury demonstrated decreased attenuation of pseudoaneurysm with no significant change in size and no active contrast extravasation. Due to hemodynamic stability and suggestion of resolving pseudoaneurysm, a nonoperative approach was maintained, with planned CT venogram one month later.

\section{Discussion}

\section{Pathophysiology and Clinical Course}

Traumatic inferior vena cava (IVC) lesions represent $30 \%$ to $40 \%$ of trauma related abdominal vascular injuries [1]. IVC aneurysms are rare clinical entity, are always acquired [2]. Most of them develop secondary to traumatic vascular injury [2] [3] [4] [5]. By definition pseudoaneurysm is a contained pulsatile hematoma near vessel wall puncture or tear that communicates with vessel [6]. Account for approximately $25 \%$ of abdominal vascular injuries [2]. The spectrum of blunt caval injuries range from intimal flaps to extensive destruction [7]. Disruption of one or more layers of the wall leads to slow extravasation of blood, allowing the areolar tissue in the vicinity to form a sac consistent with pseudoaneurysm and/weakness in the vessel wall and gradually results in dilatation and aneurysm formation [2] [4]. Though aneurysms and pseudoaneurysms of the IVC are anatomically different are discussed similarly because they behave in the same way [2].

The presentation of IVC pseudoaneurysm can vary from asymptomatic to life-threatening. Presenting symptoms include abdominal pain, IVC syndrome, tachycardia, upper gastrointestinal bleeding secondary to venobiliary fistulas, hemorrhagic shock secondary to rupture, and pulmonary embolus or other thromboembolic phenomenon.

An overall mortality rate for IVC trauma is $40 \%$ [1]. Predictors of mortality are 1) hemodynamic condition of the patient on arrival and 2) the location of the 
caval lesion [1] [2], associated injuries, preoperative lactate and base deficits, ISS and GCS [1]. Injury to suprarenal (infrahepatic) and retrohepatic segments is associated with high mortality rate, whereas injury to infrarenal segment is associated with lowest mortality rate. Active hemorrhage from injured retrohepatic IVC is frequently uncontrolled may be reason for high mortality rate.

Treatment options for IVC injury are 1) Surgical interventions 2) Endovascular techniques. The treatment strategy is based on patient's hemodynamic condition, unstable or stable with symptoms or asymptomatic. Surgical intervention treatment of choice in hemodynamically unstable patient. Endovascular methods can be used in hemodynamically stable symptomatic patients. Observation is the treatment of option in asymptomatic patients.

\section{Conclusion}

IVC pseudoaneurysm is rare clinical entity. Its clinical course depends on hemodynamic stability of patient and location of caval injury. Observation is the treatment option in hemodynamically stable, asymptomatic patients.

\section{References}

[1] Cudworth, M., Fulle, A., Ramos, J.P. and Arriagada, I. (2013) GCS as a Predictor of Mortality in Patients with Traumatic Inferior Vena Cava Injuries: A Retrospective Review of 16 Cases. World Journal of Emergency Surgery, 8, 59. https://doi.org/10.1186/1749-7922-8-59

[2] Kunkala, M., Jenkins, D.H., McEachen, J., Stockland, A. and Zielinski, M.D. (2011) Non Operative Management of Traumatic Suprahepatic Inferior Vena Cava Pseudoaneurysms. Journal of Vascular Surgery, 54, 805-825.

[3] Salour, M., Dattilo, J.B., Mingloski, P.M. and Brewer, W.H. (1998) Femoral Vein Pseudoaneurysm: Uncommon Complication of Femoral Vein Puncture.

[4] Karahan, M., Isbir, S., Baltacyoglu, F. and Caglar, M.M. (2005) False Aneurysm of the Common Femoral Vein in a Footballer. British Journal of Sports Medicine, 39, e8. https://doi.org/10.1136/bjsm.2004.012682

[5] Kim, H.O., Ji, Y.B. Lee, S.H., Jung, C. and Tae, K. (2012) Cases of Common Carotid Artery Pseudoaneurysm Treated by Stent Graft. Case Reports in Otolaryngology, 2012, Article ID: 674827. http://dx.doi.org/10.1155/2012/674827

[6] Huang, S.-W., Wei, T.-S., Liu, S.-Y. and Wang, W.-T. (2010) Spontaneous Totally Thrombosed Pseudoaneurysm Mimicking a Tendon Tear of the Wrist. Orthopedics, 33.

[7] Netto, F.A.C.S., Tien, H., Hamilton, P., Rizoli, S.B., Chu, P., Maggisano, R., Brenneman, F. and Tremblay, L.N. (2006) Diagnosis and Outcome of Blunt Caval Injuries in the Modern Trauma Center. Journal of Trauma, Injury, Infection and Critical Care, 61, 1053-1057. 